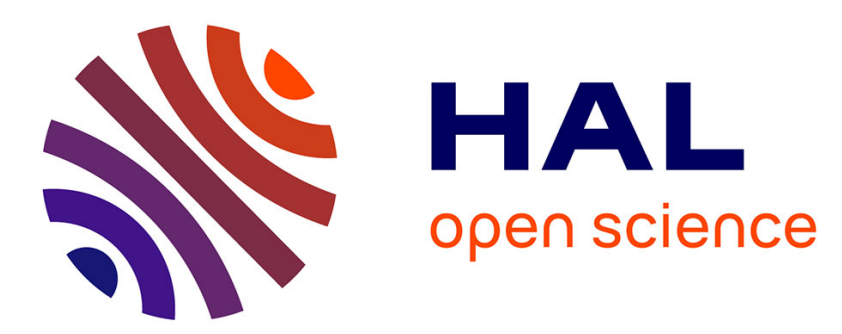

\title{
Coverage estimation of multicast OFDM systems based on stochastic geometry
}

Jose Saavedra, Christopher Flores, Anthony Busson, Pierre Duhamel

\section{To cite this version:}

Jose Saavedra, Christopher Flores, Anthony Busson, Pierre Duhamel. Coverage estimation of multicast OFDM systems based on stochastic geometry. IEEE ICA/ACCA, Oct 2018, Concepcion, Chile. hal-01858575

\section{HAL Id: hal-01858575 \\ https://hal-centralesupelec.archives-ouvertes.fr/hal-01858575}

Submitted on 21 Aug 2018

HAL is a multi-disciplinary open access archive for the deposit and dissemination of scientific research documents, whether they are published or not. The documents may come from teaching and research institutions in France or abroad, or from public or private research centers.
L'archive ouverte pluridisciplinaire HAL, est destinée au dépôt et à la diffusion de documents scientifiques de niveau recherche, publiés ou non, émanant des établissements d'enseignement et de recherche français ou étrangers, des laboratoires publics ou privés. 


\title{
Coverage estimation of multicast OFDM systems based on stochastic geometry
}

\author{
José Saavedra, Christopher Flores, Anthony Busson, Pierre Duhamel
}

\begin{abstract}
Quality of service has become crucial in wireless communications, due to the high data rates needed to provide multimedia services with sufficient quality. In this sense, the integration of OFDM in wireless technologies has made it possible to grant reliable communications, reducing the effects of attenuation and multipath propagation that degrades the services in environments where the devices are located. This problem may become even more important if, as it is expected, the classical broadcast TV is left to the wireless network operators, in order to recover the frequencies that are currently used for this service. This paper aims to provide efficient tools to estimate the coverage of an OFDM transmission system using stochastic geometry and information theory to model the capacity of a cell when users are known to the transmitter (multicast situation). We consider that users are distributed uniformly over a fixed radius ball from the base stations. We derive the equations and develop simulations to model the distribution of channel capacity to ensure a certain quality of service. Our results indicate that for a user, on average, wireless multicast can be improved by $20 \%$ compared to classical broadcast.
\end{abstract}

Keywords-Wireless Multicast, OFDM, Coverage Capacity.

\section{INTRODUCTION}

I $\mathrm{N}$ the last decade wireless technologies have grown quickly. The devices that deliver telephony, data and multiple services, are widely deployed. Between the standards, Orthogonal Frequency Division Multiplexing (OFDM) are more and more present. By the side of wireless packet data transmission we have $802.11 \mathrm{a} / \mathrm{g} / \mathrm{n} / \mathrm{ac} / \mathrm{ad}$ [1], popularly known as Wireless Fidelity (WI-FI) and by the side of mobile communications we have the consortium of the 3rd Generation Partnership Project Long Term Evolution (3GPP-LTE) with 5G [2] [3], both have integrated OFDM given its capacity to overcome impairments due to scattering environments where the devices are deployed. The main trick of OFDM is to take a channel bandwidth and split them in many orthogonal sub-channels with low data rate, granting reliable communication.

Usually, in wireless networks, when the same data stream is provided to a group of users, Time Division Multiplexing is used to allocate the same data to different block time resources, for each user, with power allocation and the modulation and coding scheme negotiated between the base station (BS) and the user device. This is very costly in terms of resource (one

J. Saavedra is with the Departamento de Medio Ambiente y Energía, Universidad Católica de la Santísima Concepción, Concepción, Chile.

C. Flores is with the Departamento de Ingeniería Eléctrica, Universidad de Concepción, Concepción, Chile.

A. Busson is with the Laboratory of Parallel Computing, ENS - CNRS Inria, University of Lyon 1,Villeurbanne, France.

P. Duhamel is with the L2S - CNRS-CentraleSupelec-Universite Paris-Sud, Gif-sur-Yvette, France. resource allocated to each user). In classical broadcasting, in contrast, the same information is transmitted via the same resource to all users, which are a priori unknown to the transmitter. This corresponds to a large saving in terms of resource but no power allocation can be performed by the transmitter, which corresponds to a waste of energy. This strategy is unavoidable when the number of users is very large. However, there is a possibility that, in the near future, pure broadcast (for terrestrial TV) will disappear, and the wireless networks will be in charge of performing this service [4] [5]. If this happens, the number of users to be served simultaneously by the same transmitter (base station) will be reasonable, and one can envision to use a wireless multicast strategy, where the users are known to the transmitter, which can therefore perform some optimization. The frontier between these two cases is however not obvious, and this paper tries to clarify this trade-off.

Our work focuses on find an objective criterion to decide if the optimization is still useful, or if the uniform power allocation is the best way to transmit data to a group of users. We name wireless multicast when a group of users subscribe to the same service and the CSI are available on BS. When the uniform power allocation is used to transmit the same data, we name it broadcast, like the old-fashioned broadcast-TV.

In such a situation the transmitter has no hope that the transmitted signal will be received by all users, because the randomness of channels makes some of them really intractable. In such case, one way to take these events into account is via outage capacity: a broadcast channel is always designed in such a way that $x \%$ of the users are certain to obtain a given rate [6] [7].

In practical situations, the coverage of an access point or base station can be made using long simulations using a merge between analytic and empirical methods [8]. However, in the last decade, techniques based on stochastic geometry [9] [10] were proposed, which allow to solve analytically such problems related to wireless communications.

Stochastic geometry (SG) is a discipline of applied mathematics that studies spatial random patterns with emphasis on the theory of Point Processes (PP) [10]. The classic SG models are mainly based on the study of Poisson Point Processes (PPP) [11]. The simplest model is the Boolean (BM), which is composed of random balls centered at each point of the process [10]. Another model commonly used in SG are the Voronoi Tessellations (VT), which are composed of finite polyhedrons (cells) containing all the points that are closest to the center of the cell [10] [12]. The SG has diverse applications in biology, astronomy, wireless communications, among others. 
For instance, in the context of wireless communications, the location of base stations (BSs) can be modeled using a PPP, while coverage areas can be defined using a Voronoi cell [13].

We intend to extend the stochastic geometry techniques to OFDM channels systems, and it will be shown that analytical solutions are not fully feasible, and that only mixed analyti$\mathrm{cal} /$ numerical solutions are obtained. This process will anyway be faster than the many simulations usually required.

It's known that the percentage of users meeting the given bitrate constraint depend on their location. Therefore, we assume that the users are distributed according to a certain spatial point process. It allows us to consider the spatial distribution of the users around the base station,from which we deduce the distribution of the capacity. Then, for a typical user, we compute a coverage area where a minimum/fixed capacity is guaranteed with a certain probability. This corresponds to an outage probability on the capacity. This outage probability may also be interpreted as the proportion of users for which this fixed capacity will be offered, which is precisely the desired design criterion for old-fashioned broadcast transmission.

Our solution involves some approximations to obtain semianalytical results, therefore, a first set of numerical results checks that the different approximations of the distributions (channels, capacity of a given channel, etc.) involved in the computation of the final capacity are accurate. Then, the extrapolated capacity is compared to simulations, in order to check if our optimization is correct over this scenario, meaning that the required percentage of users indeed meets the desired bit rate.

\section{SySTEM MODEL}

Our model consider an ideal OFDM system were the channels are perfectly orthogonal. The user $\ell$ is one user among $L$-total users. The received signal of the user $\ell$ is:

$$
\mathbf{y}_{\ell}=\mathbf{H}_{\ell} \mathbf{x}+\mathbf{z}_{\ell}
$$

where $\mathbf{x}=\left(x^{(1)}, . ., x^{(N)}\right) \sim \mathbb{C} \mathcal{N}\left(0, S_{\mathbf{x}}\right)$ is the input vector of $N$ OFDM symbols and $S_{\mathbf{x}}$ is the covariance matrix of the vector $\mathbf{x}$. Since the modulated symbols are independent and orthogonal, the covariance matrix $S_{\mathbf{x}}$ is a diagonal matrix such that $S_{\mathbf{x}}=\operatorname{diag}\left\{p_{n}\right\}, n \in\{1, \ldots, N\}$, where $p_{n}$ is the power allocated to the $n$-th subcarrier. The total power available to optimize is $P$, such that $\sum_{n=1}^{N} p_{n} \leq P$. The Matrix $\mathbf{H}_{\ell}$ is the a diagonal channel gain matrix which dimension is $N \times N$, the elements are the channel impulse response of channel on each orthogonal sub-carrier frequency, $\left.\mathbf{H}_{\ell}=\operatorname{diag}\left\{h^{(\ell, n)}\right\}\right)$ and is the Channel Sate Information (CSI) present at the transmitter. The vector $\mathbf{z}$ is the $\mathrm{AWGN}$ at the receiver $\mathbf{z}_{\ell} \sim \mathbb{C} \mathcal{N}\left(0, S_{\mathbf{z}}\right)$. The problem of broadcast the same information for $L$-users are faced in [14]. Where we develop an optimal algorithm to allocate efficiently the power among the subcarriers. From that we recall the capacity of one user $(L=1)$, since we use it to perform a coverage statistic over a spatial distribution. And we can compare with previous results.

We recall the capacity formula for the ideal OFDM scheme, as parallel independent channel capacity [14] [15].

$$
C=\frac{1}{N} \sum_{n=1}^{N} \log \left(1+\frac{\left|h^{(n)}\right|^{2} p_{n}}{\sigma_{z}^{2}}\right) \quad[\text { bits } / s / H z]
$$

The complex subcarrier gain $h^{(n)}$ is expressed in the frequency domain.

\section{OFDM REALISTIC CHANNEL AND COVERAGE MODELING}

The goal is to find a closed-form expression to calculate the coverage, and have an impartial instrument to say if the multicast optimization is still useful under a spatial distribution of the users, with an outage criterion in mind.

Let $P\left(C \geq C_{\text {out }}\right)$ denote the outage probability. It is the probability that the capacity of a typical user, denoted $C$, is greater than a predetermined value $C_{\text {out }}$ [7], [15]. Given a capacity requirement $C_{\text {out }}$ and an outage probability, we can calculate the maximal coverage, i.e. the maximal distance where users can be distributed while statistically meeting these two constraints.

\section{A. Realistic Channel}

The first step towards characterizing properly the capacity is to first evaluate the probability density function of the channel $h^{(n)}$. We know that [8] [1] it is modelled by (3), evaluated at frequency $f_{n}$ :

$$
h^{(n)}=\sum_{i=1}^{I} \beta_{i} e^{-j\left(2 \pi f_{n} \tau_{i}+\theta_{i}\right)}
$$

Where $\beta_{i}$ is the product of the path loss gain $\sqrt{\frac{1}{L(D)}}$ and the complex gain of the path $g_{i}: \beta_{i}=\sqrt{\frac{1}{L(d)}} \cdot g_{i}$. We can express it as:

$$
h^{(n)}=\sqrt{\frac{1}{L(D)}} \sum_{i=1}^{I} g_{i} e^{-j\left(2 \pi f_{n} \tau_{i}+\theta_{i}\right)}
$$

The multipath part of this expression can be written as:

$$
g^{(n)}=\sum_{i=1}^{I} g_{i} e^{-j\left(2 \pi f_{n} \tau_{i}+\theta_{i}\right)}
$$

Given that the path gains $\left\{g_{i}\right\}$ are Rayleigh distributed with parameter $\sigma_{i}$ and that the phases $\left\{\theta_{i}\right\}$ are uniformly distributed on the interval $[0,2 \pi]$, we define $\bar{g}_{i}=g_{i} e^{-j \theta_{i}}$ as a single random variable with a complex gaussian distribution with zero mean and variance $\sigma_{i}^{2}$. The random variables $\left\{\bar{g}_{i}\right\}_{i=1, \ldots, I}$ form a sequence of independent complex Gaussian random variables with zero mean and different variances. The real and imaginary parts are assumed to be independent. The multipath part thus reduces to:

$$
g^{(n)}=\sum_{i=1}^{I} \bar{g}_{i} e^{-j 2 \pi f_{n} \tau_{i}}
$$

Finally the complex channel gain $h^{(n)}$ can be viewed as: 


$$
h^{(n)}=\sqrt{\frac{1}{L(D)}} g^{(n)}
$$

But we can see that $g^{(n)}$ is a combination of different random variables, all of them with zero mean, but of various variances. Under this situation the central limit theorem does not apply, because the involved random variables have different variances. However, in order to simplify our next computations, we will make a Gaussian approximation of $g^{(n)}$. In fact, for the considered model, the model $E$ described in [16], we can empirically show by simulation that the real and the imaginary part of $g^{(n)}$ are very close to Gaussian distributions. We illustrate in Fig, 1 the distribution of imaginary and the real part of $g^{(n)}$ for this model. In this figure we can appreciate the actual model in red line and the approximated by the complex gaussian function in cross blue line. The curves are almost superimposed, and the complex normal distribution seems to be a good approximation for our purpose.

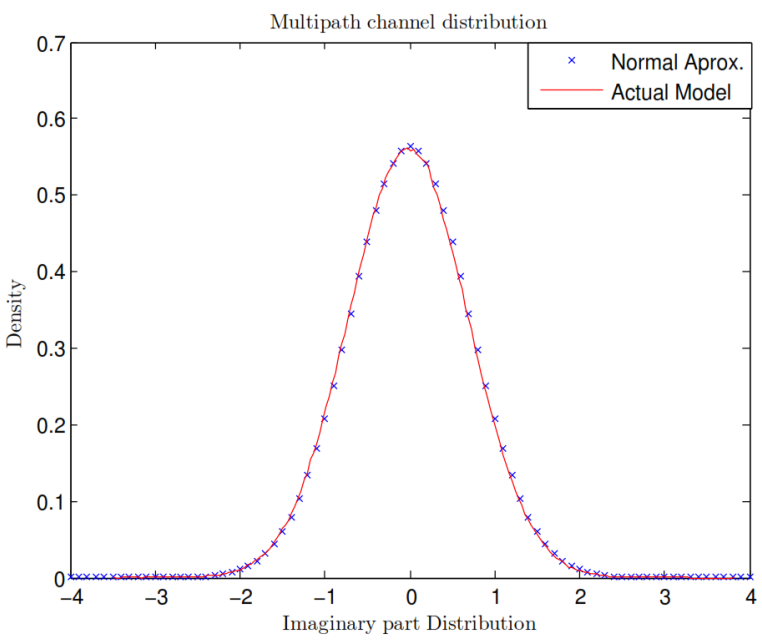

(a) Imaginary Multipath Distribution

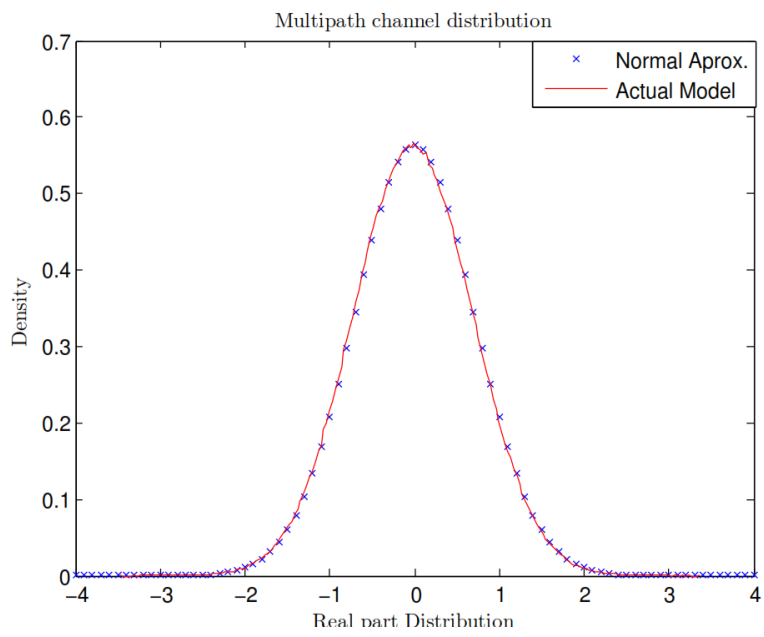

(b) Real Multipath Distribution

Fig. 1: Multipath Channel Distribution

Therefore, in the rest of this work, we assume that the random variables $\left(g^{(n)}\right)_{n}$ follow complex normal distributions.
Hence, the probability distribution function of $g^{(n)}$ is approximated by:

$$
f_{g^{(n)}}(z)=\frac{1}{\pi \sum_{i=1}^{I} \sigma_{i}^{2}} \exp \left\{-\frac{\bar{z} z}{\sum_{i=1}^{I} \sigma_{i}^{2}}\right\}
$$

which will be useful for a proper characterization of the capacity distribution.

\section{B. Outage probability}

Unfortunately, analytical computation of the capacity pdf is not tractable for our model. Instead, we approximate it with a known distribution. Parameters of this distribution will be deduced from mean and variance of the capacity. Therefore, in this Section, we compute mean and variance of the capacity for our model.

If we retake the formula (2) and put inside the channel expression (7) we obtain:

$$
C=\frac{1}{N} \sum_{n=1}^{N} \log \left(1+\frac{1}{L(D)}\left|g^{(n)}\right|^{2} \frac{p_{n}}{\sigma_{z}^{2}}\right) \quad[\text { bits } / s / H z]
$$

The random variables that compose the equation of the capacity (9) are the distance $D$ and the complex channel gains $g^{(n)}$.

The distance $D$ is the distance between a typical user and the base station. We assume that users are distributed in an observation window. In our numerical evaluation, it consists in a ball with radius $R$. Within this ball, users location can follow any distribution. Here we consider that users are independently and uniformly distributed in this window. Since the base station is at the center of this ball, the pdf of the distance from a user to the BS is given by:

$$
f_{D}(d)=\frac{2 d}{R^{2}}
$$

where $d$ belongs to $[0, R]$.

The multipath complex channel gain $g^{(n)}$ has $f_{g^{(n)}}(z)$ as probability density function (8). In order to characterize the capacity random variable, we start with an expression of the expectation considering the cited distributions and we follow with the variance.

The computation of the mean capacity is straightforward. It is given by:

$$
\begin{gathered}
\mathbb{E}[C]=\frac{1}{N} \sum_{n=1}^{N} \mathbb{E}\left[\log _{2}\left(1+\frac{1}{L(D)}\left|g^{(n)}\right|^{2} \frac{p_{n}}{\sigma_{z}^{2}}\right)\right] \\
=\frac{1}{N} \sum_{n=1}^{N} \int_{0}^{R} \int_{\mathbb{C}} \log _{2}\left(1+\frac{1}{L(d)}|z|^{2} \frac{p_{n}}{\sigma_{z}^{2}}\right) \ldots \\
\ldots f_{g^{(n)}}(z) f_{D}(d) d z d d
\end{gathered}
$$

For the variance, we get:

$$
\operatorname{Var}(C)=\sum_{n=1}^{N} \operatorname{Var}\left(C_{n}\right)+2 \sum_{n=1, i>n}^{N-1, N} \operatorname{Cov}\left(C_{i}, C_{n}\right)
$$

where $C_{n}$ is the capacity over one subcarrier.

Considering that the channels can have a correlation between the subcarriers, the covariances are not nil. This correlation is due to the random variables $\bar{g}_{i}$ (see formula 6) which are the same for all the channels. 
The covariance $\operatorname{Cov}\left(C_{i}, C_{n}\right)$ has the form:

$$
\begin{gathered}
\operatorname{Cov}\left(C_{i}, C_{n}\right)=\int_{0}^{R} \int_{\mathbb{C}} \int_{\mathbb{C}} c_{n}\left(z^{n}, d\right) c_{i}\left(z^{i}, d\right) \ldots \\
\quad \ldots f_{g^{(n)}, g^{(i)}}\left(z^{(n)}, z^{(i)}\right) f_{D}(d) d z^{(n)} d z^{(i)} d d
\end{gathered}
$$

where $c_{n}\left(z^{n}, d\right)$ is the capacity of the subcarrier $n$ for a given sample of $g^{n}$ (equals to $z^{n}$ ) and a sample of the distance $D(d) . f_{\left(g^{(n)}, g^{(i)}\right)}\left(z^{(n)}, z^{(i)}\right)$ is the joint probability density function of the random variables $\left(g^{n}, g^{i}\right)$.

If we assume that the vector $\left(g^{(n)}, g^{(i)}\right)$ is still normal complex, the joint distribution is:

$$
\begin{gathered}
f_{\left(g^{(n)}, g^{(i)}\right)}\left(z^{(n)}, z^{(i)}\right)=\frac{1}{\pi^{2} \operatorname{det}(\Gamma)} \cdots \\
\exp \left\{\frac{\sum_{i=1}^{I} \sigma_{i}^{2}}{\operatorname{det}(\Gamma)}\left(z^{(n)} \overline{z^{(n)}}+z^{(i)} \overline{z^{(i)}}\right)-2 \operatorname{Re}\left(\gamma_{n, i} z^{(i)} \overline{z^{(n)}}\right)\right\} \\
\text { with } \operatorname{det}(\Gamma)=\left(\sum_{i=1}^{I} \sigma_{i}^{2}\right)^{2}-\left|\gamma_{n, i}\right|^{2} \text { and } \\
\gamma_{n, i}=\sum_{u=1}^{l} \sigma_{u}^{2} \exp \left\{-j 2 \pi\left(f_{n}-f_{i}\right) \tau_{u}\right\}
\end{gathered}
$$

The joint distribution above is obtained under the assumption that $\left(g^{(n)}, g^{(i)}\right)$ is normal complex, and is function of variance, covariance of these two random variables [17], [18].

It can be seen that the mean and the variance do not have an easy form. These integrals must be calculated numerically.

\section{Modelling the capacity pdf}

Let $f(E[C], \operatorname{Var}[C], x)$, the pdf parameterized with the mean $E[C]$ and the variance $\operatorname{Var}[C]$. To calculate the outage probability under the given scenario, we use the following formula:

$$
\begin{aligned}
\mathbb{P}\left(C \geq C_{\text {out }}\right) & =1-\mathbb{P}\left(C \leq C_{\text {out }}\right) \\
& =1-\int_{0}^{C_{\text {out }}} f(E[C], \operatorname{Var}[C], x) d x
\end{aligned}
$$

Once the mean and variance are obtained, we will compare the shape of the actual pdf obtained by simulation with a well known function, in order to find the best fit. Good candidates belong to the log-normal probability distributions.

For instance $f(E[C], \operatorname{Var}[C], x)$ can be the log-normal pdf. It can be used to approach the empirical pdf of the capacity under a realistic environment. This function has the following form:

$$
\begin{gathered}
f(E[C], \operatorname{Var}[C], x)=\frac{1}{x \sigma_{V}} \exp -\left(\frac{\left(\ln (x)-\mu_{E}\right)^{2}}{2 \sigma_{V}}\right) \\
\text { Where } \mu_{E}=\ln \left(\frac{E[C]^{2}}{\sqrt{\left(\operatorname{Var}[C]+E[C]^{2}\right)}}\right) \\
\sigma_{V}=\ln \left(\frac{\operatorname{Var}[C]}{E[C]^{2}}+1\right)
\end{gathered}
$$

where the parameters $\mu_{E}$ and $\sigma_{V}$ are deduced from mean and variance of the capacity $C$. Once we have (17) parameterized, we can calculate numerically $C_{\text {out }}$ from (16). The mean $E[C]$ and the $\operatorname{Var}[C]$ was calculated using numerical integration. We took the probabilistic distribution (10) with $R=200$. We use the parameters of the model $\mathrm{E}$ [16] to model the pdf of the fading channel, and parameterize (8). For the path loss, we use the equations from [14], taking in account the distance breakpoint. Using this parameters, the calculated mean has value: $E[C]=0.4529$ and the variance: $\operatorname{Var}[C]=0.8827$. On the other hand, in order to have values to compare, we perform an empirical test generating samples, the empirical mean are 0.4525 and the variance of the samples are 0.9996 . The difference are essentially due to the iterations needed to perform the integrations.

With that the log-normal distribution are parameterized and the pdf is shown in the Fig. 2. The curve in blue is the pdf of the empirical random variable, made with many samples. The curve in magenta, is the log-normal pdf but parameterized with the empirical values of the mean and variances of the samples. And finally, the black curve shown is parameterized with the computed values using the theoretical pdf. It appears that the curves are quite different on the peak of the distributions, but the distribution tails are very close to each other.

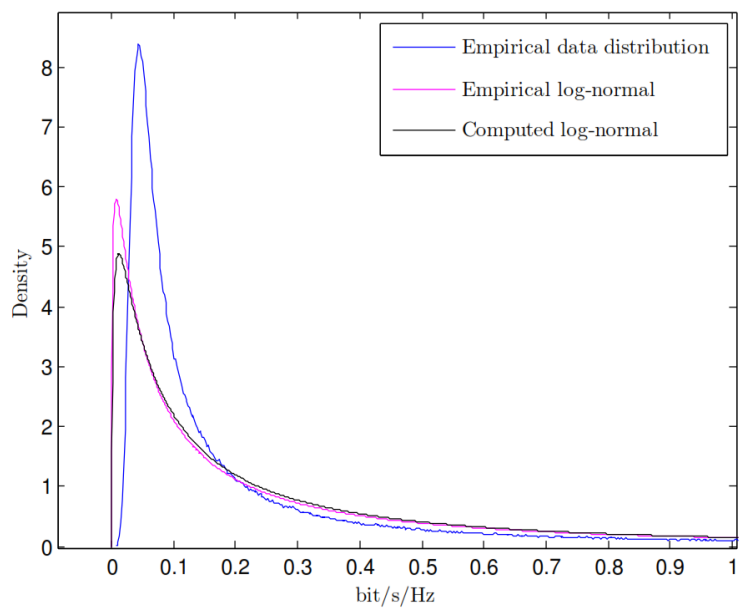

Fig. 2: PDF of the capacity of a given scenario

The formula (16) can be used in different ways. It gives the distribution of the capacity offers to broadcast communications when the parameters are given. It can also be used to parameterize the system in order to achieve a certain quality of service: fix the maximum radio range $\mathrm{R}$ of the BS to ensure a capacity $C_{\text {out }}$ with a certain probability, etc. In the next section we show numerical results that can be found performing many realizations.

\section{NUMERICAL RESULTS}

The simulations are performed under the assumption that the users are uniformly distributed over a ball of radius $R=200[m]$. To aim a SNR close to $10 d B$ we set the noise with a power of $1.0 \times 10^{-12}[W]$. And to aim a SNR close to $15 \mathrm{~dB}$ we set the noise with a power of $1.0 \times 10^{-13}[\mathrm{~W}]$. We perform the simulations using our optimization algorithm for both cases and compare with the non optimized case. We set the $\mathbb{P}\left(C \geq C_{\text {out }}\right)=0.95$ and we calculate $C_{\text {out }}$. The target here is to check how the optimization criterion (sum capacity) 
matches the broadcast optimization criterion (outage capacity) . Also we see if the extrapolation by a known pdf are useful in order to establish a design criteria for the broadcast channel situation.

\section{A. Numerical results at $10 \mathrm{~dB}$}

Fig. 3 shows the results and present the followed concepts:

- The capacity outage $C_{\text {out }}$ where the probability is $95 \%$ for Broadcast scheme, Brown - Circle.

- The capacity outage $C_{\text {out }}$ where the probability is $95 \%$ for Optimized scheme, Dark Red - Star.

- The Mean Broadcast capacity where probability is over $95 \%$, Cyan - Cross.

- The Mean Optimized capacity where probability is over $95 \%$, Pink - Square.

- The Mean of the Broadcast capacity (100\%), Yellow triangle.

- The Mean of the Optimized capacity (100\%), blue Diamond.

- Log-Normal based computed Outage Capacity, BlueGreen - Plus.

- Log-Normal based empirical Outage Capacity, Red Dash.

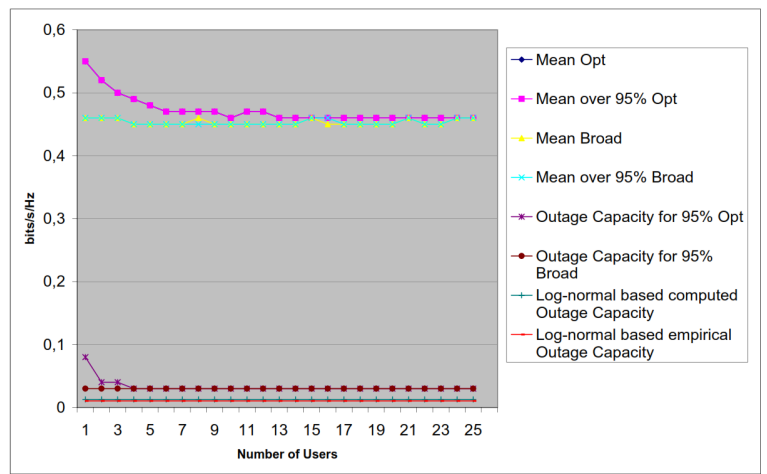

Fig. 3: Mean capacity, Outage capacity for $95 \%$ and Mean over $95 \%$ for SNR $10 \mathrm{~dB}$

At first glance we can see that the capacity gain in mean is weak. From the Fig. 3, we can see $20 \%$ of increase in one user and $13 \%$ when we have 2 users. The mean of the sub-set that is over $95 \%$ of probability is equal with the one with all the samples. The mean of the optimized capacity shows weak improvement between one and eight users. The outage capacity of the samples is near $0.03[$ bits $/ \mathrm{s} / \mathrm{Hz}]$. We try to approximate the actual pdf by a log normal pdf. When we parameterize the log-normal pdf with the mean and the variance of the samples, the log-normal based empirical outage capacity is $0.0103[\mathrm{bits} / \mathrm{s} / \mathrm{Hz}]$. And when we parameterize with the mean and the variance obtained computing $E[C]$ and $\operatorname{Var}[C]$, the log-normal based computed capacity is $0.0127[$ bits $/ \mathrm{s} / \mathrm{Hz}]$.

On the other hand, we consider the percentage increase histograms (Fig. 4). It is computed for each sample as:

$$
\left(\frac{\text { Optimized Capacity }}{\text { Broadcast Capacity }}-1\right) \cdot 100
$$

Fig. 4 shows the distribution of this percentage increase. A percentage increase of $0 \%$ means that the optimized and broadcast capacity are the same (the ratio is 1 ).

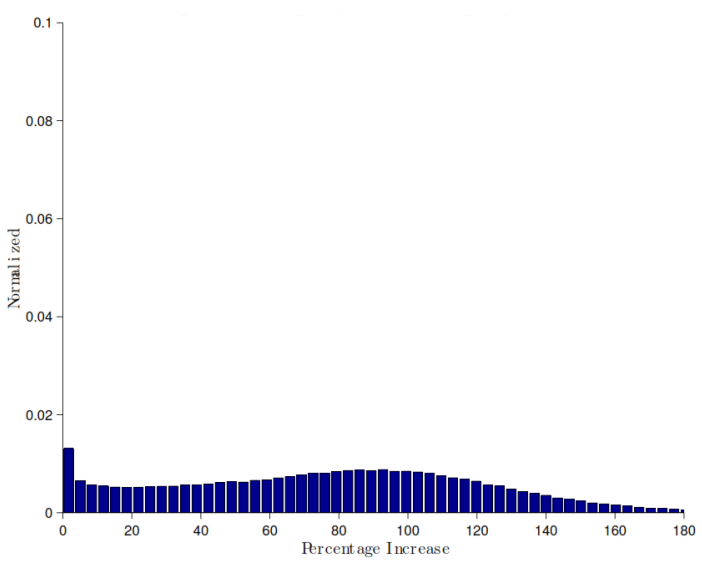

(a) 1 User

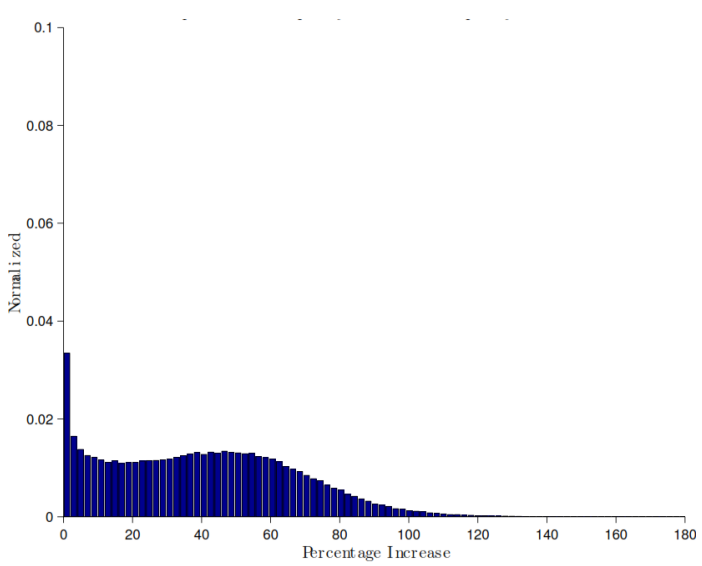

(b) 2 Users

Fig. 4: Percentage increase at $10 \mathrm{~dB}$

We can observe that for approximately $30 \%$ of the samples, there is a percentage increase superior to $100 \%$, meaning that the optimized capacity is at least two times greater than the broadcast one. Also, it is worth noting that for one user the mean percentage increase is $72 \%$. The difference with the results shown in Fig. 3 may be explained by the fact that these improvements mainly concerns users with small capacity keeping mean capacity quite constant. The later can be appreciated comparing the histograms in Fig. 5, for one user. It clearly appears that the distribution of the capacity is different for the first part (approximately for capacity less than $0.5 \mathrm{bit} / \mathrm{s} / \mathrm{Hz}$ ), and similar for higher capacities. More precisely, for one user the optimized mean capacity is $0.55 \mathrm{bits} / \mathrm{s} / \mathrm{Hz}$, the broadcast mean capacity is $0.46 \mathrm{bits} / \mathrm{s} / \mathrm{Hz}$. If we compare the means we have $20 \%$ of increase between the means. For 2, 3 , 4 and 5 users this comparison gives $13 \%, 8.7 \%, 8.8 \%$ and $6.66 \%$ respectively.

\section{CONCLUSION}

In this work we explore the capacity of the OFDM with new elements. We consider that the user are distributed uniformly over a ball with a fixed radius from the base station. Following 


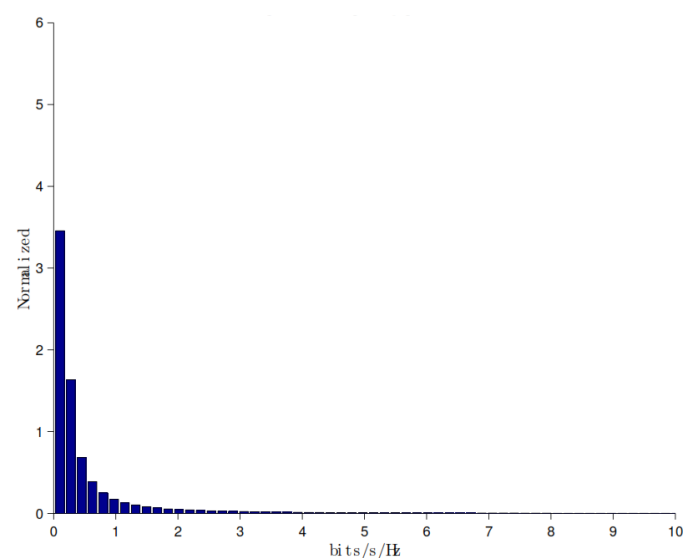

(a) Optimized capacity one user

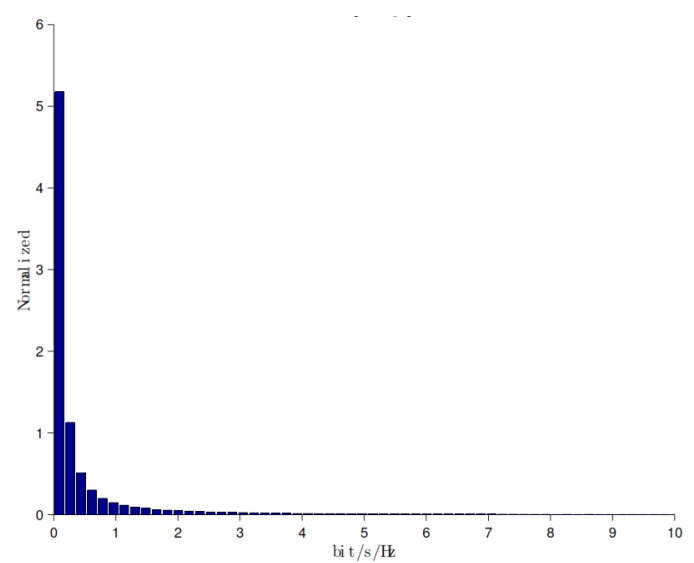

(b) Broadcast capacity one user

Fig. 5: Optimized and Broadcast Capacity, one user at $10 \mathrm{~dB}$

the goal of characterizing the outage probability, we derive expressions for the mean and the variance of the capacity under the studied scenario. These two quantities could be used to extrapolate the capacity distribution. The derived expression are difficult to handle and finally we perform numerical simulations. From this distribution, the broadcast can be designed in order to ensure a certain quality of service. For example, the system can be tuned in order to ensure to a certain proportion of users and in a certain radio range a given capacity. Comparisons between the optimized and the classical broadcasts have shown that there is not a significant improvement in average. The mean capacity increases of 20 $\%$ for one user and decreases slowly with the number of users. But, it concerns users with small capacity that explains the poor improvement in average. However, this is an issue of practical importance, since the largest improvement is provided to the users with the smallest quality of service. A topic that could extend this work would consist in comparing the capacity improvement with different spatial distributions. Indeed, it appeared clearly that the distance between the users and the BS impacts the capacity gain of the optimized case. Spatial distributions where the users are far from the BS should greatly increase the benefit of an optimized allocation power, whereas distributions where the users are gathered near the BS should lead to cases where this optimization is almost useless. It could be particularly interesting to collect real users spatial distributions and real scenarios, to have better insight of the situations where multicast is really worth the additional complexity needed for the optimization.

\section{ACKNOWLEDGMENT}

The authors would like to thank to Dirección de Investigación e Innovación from Universidad Católica de la Santísima Concepción (INDIN Grant \# 0317) for their support to this research.

\section{REFERENCES}

[1] E. Perahia and R. Stacey, Next Generation Wireless LANs: Throughput, Robustness, and Reliability in 802.11n. Cambridge University Press, 2008.

[2] V. Wong, R. Schober, D. Ng, and L. Wang, Key Technologies for $5 G$ Wireless Systems, ser. Key Technologies for 5G Wireless Systems. Cambridge University Press, 2017. [Online]. Available: https://books.google.cl/books?id=HdAoDwAAQBAJ

[3] C. X. Wang, F. Haider, X. Gao, X. H. You, Y. Yang, D. Yuan, H. M. Aggoune, H. Haas, S. Fletcher, and E. Hepsaydir, "Cellular architecture and key technologies for $5 \mathrm{~g}$ wireless communication networks," IEEE Communications Magazine, vol. 52, no. 2, pp. 122-130, February 2014.

[4] S. Nakahara, M. Okano, M. Takada, and T. Kuroda, "Digital transmission scheme for isdb-t and reception characteristics of digital terrestrial television broadcasting system in japan," IEEE Trans. on Consum. Electron., vol. 45, no. 3, pp. 563-570, Aug. 1999. [Online]. Available: http://dx.doi.org/10.1109/30.793541

[5] E. T. S. Institute, Digital Video Broadcasting (DVB): Implementation guide for DVB terrestrial services; transmission aspects, European Telecommunications Standards Institute Std. ETSI-TR-101, 2007. [Online]. Available: http://www.etsi.org

[6] L. H. Ozarow, S. Shamai, and A. D. Wyner, "Information theoretic considerations for cellular mobile radio," vol. 43, no. 2, pp. 359-378, 1994.

[7] A. Goldsmith, Wireless Communications. Cambridge University Press, Sep. 2005

[8] T. Rappaport, Wireless Communications: Principles and Practice, 2nd ed. Upper Saddle River, NJ, USA: Prentice Hall PTR, 2002.

[9] D. Stoyan, W. S. Kendall, and J. Mecke, Introduction to Stochastic Geometry, Wiley, Ed. John Wiley \& Sons, 1987.

[10] F. Baccelli and B. Błaszczyszyn, "Stochastic geometry and wireless networks: Volume i: Theory," Foundations and Trends $®$ in Networking, vol. 3, no. 3-4, pp. 249-449, 2009.

[11] P. Calka, D. Coupier, J.-B. Gouéré, A. Rousselle, and A. Vergne, "Stochastic geometry: Boolean model and random geometric graphs," ESAIM: Proceedings and Surveys, vol. 51, pp. 175-192, 2015.

[12] H. H. Yang and T. Q. S. Quek, "Sir coverage analysis in cellular networks with temporal traffic: A stochastic geometry approach," Available as arXiv:1612.01276, 2018.

[13] M. A. Klatt, G. Last, K. Mecke, C. Redenbach, F. M. Schaller, and G. E. Schröder-Turk, "Cell shape analysis of random tessellations based on minkowski tensors," in Tensor Valuations and Their Applications in Stochastic Geometry and Imaging. Springer, 2017, pp. 385-421.

[14] J. Saavedra and P. Duhamel, "Multicast or broadcast in multiuser ofdm systems," in Wireless Communications and Signal Processing (WCSP), 2011 International Conference on. IEEE, 2011, pp. 1-5.

[15] D. Tse and P. Viswanath, Fundamentals of Wireless Communications. New York, NY, USA: Cambridge University Press, 2005.

[16] J. Medbo and P. Schramm, Channel models for HIPERLAN/2 in different indoor scenarios, ETSI BRAN Std. 3ERI085B, 1998.

[17] A. van den Bos, "The multivariate complex normal distribution-a generalization," IEEE Transactions on Information Theory, vol. 41, no. 2, pp. 537-539, Mar. 1995. [Online]. Available: http://dx.doi.org/ 10.1109/18.370165

[18] B. Picinbono, "Second-order complex random vectors and normal distributions," IEEE Transactions on Signal Processing, vol. 44, no. 10, pp. 2637-2640, Oct. 1996. [Online]. Available: http: //dx.doi.org/10.1109/78.539051 